\title{
Mesophase behaviour of polyhedral particles
}

\section{Umang Agarwal and Fernando A. Escobedo*}

School of Chemical and Biomolecular Engineering, Cornell University, Ithaca, NY 14853

* Correspondence should be addressed to F.A.E., e-mail: fe13@cornell.edu 
Translational and orientational excluded-volume fields encoded in particles with anisotropic shapes can lead to purely entropy-driven assembly of morphologies with specific order and symmetry. To elucidate this complex correlation, we performed detailed Monte Carlo simulations of six convex space-filling polyhedrons, namely, truncated octahedrons, rhombic dodecahedrons, hexagonal prisms, cubes, gyrobifastigiums, and triangular prisms. Simulations predict the formation of various novel liquid-crystalline and plastic-crystalline phases at intermediate volume fractions. By correlating these findings with particle anisotropy and rotational symmetry, simple guidelines for predicting phase behaviour of polyhedral particles are proposed: high rotational symmetry is in general conducive to mesophase formation, with low anisotropy favouring plastic-solid behaviour and intermediate anisotropy (or high uniaxial anisotropy) favouring liquid crystalline behaviour. It is also found that dynamical disorder is crucial in defining mesophase behaviour, and that the apparent kinetic barrier for the liquid-mesophase transition is much lower for liquid crystals (orientational order) than for plastic solids (translational order).

Currently a major focus in material science is to engineer particles with anisotropic shapes and interaction fields which can be self-assembled into target complex structures $^{1,2}$. Assemblies of anisotropic particles undergo order-disorder transitions involving changes in both translational and orientational degrees of freedom and can lead to phases with partial structural order or "mesophases"3,4 These ordered assemblies have distinctive electronic, optical, and dynamical properties ${ }^{4,5}$ and are 
highly desirable for fabrication of advanced electronic, photonic, and rheological devices $^{6,7}$. Although numerous theoretical ${ }^{8-13}$ and experimental ${ }^{14,15}$ studies on mesophase behaviour of particles with anisotropic shapes have been reported, a roadmap marking out the most probable mesophases that could be formed by constituent particles with particular geometrical features is still incomplete. Exploring such relations will translate into a deeper understanding of the phase behaviour of colloidal systems with different particle shapes; e.g., Jiao et al. ${ }^{16}$ reported that unlike ellipsoids, convex superballs can be optimally packed in dense Bravais lattices because they are less symmetric but more isotropic than the former. Similarly, Torquato et al. ${ }^{17}$ recently conjectured that amongst all Platonic and Archimedean solids, only those having central symmetry can pack into Bravais lattices.

The present study focuses on an important and well defined set of shapes, viz., regular space-filling polyhedrons (SFPs) ${ }^{18}$ which has only 5 convex members, namely: truncated octahedron, hexagonal prism, cube, triangular prism, and gyrobifastigium. Note that while non-SFPs (like tetrahedra) may have a non-trivial solid ordering and densest packing ${ }^{19,20}$, SFPs have the advantage of always having a priori known, perfect crystalline order. The key question this work addresses is depicted in Fig. 1a: how is the SFP solid order lost as the concentration decreases? Rather than losing all order at once (in a crystal-to-isotropic transition), we hypothesized that disordering would likely happen in stages, giving rise to "meso-phases" where, e.g., only orientational order is lost (as in plastic or rotator crystals) or just positional order is lost (as in liquid crystals). More than one intermediate mesophase could also be observed. Note also that 
mesophases are expected to lower the isotropic-to-crystal barrier and hence serve as conduits that catalyze the assembly of crystalline order from isotropic states. An approximate roadmap illustrating the various possibilities is presented in Fig. 1b, which will be discussed in full detail later in the article. Briefly, we identified two key parameters, asphericity ${ }^{17}$ of the particles (measure of anisotropy) and orientational symmetry ${ }^{21,22}$ as global descriptors of these particle fields, and show that their relative values are good indicators to predict mesophase behaviour.

The six shapes used in this study are cubes (Platonic solid), truncated octahedrons "TO" (Archimedean solid), rhombic dodecahedrons "RD" (Catalan Solid), gyrobifastigiums "GB" (Johnson solid), hexagonal prisms "HP" and triangular prisms "TP" (prisms and antiprisms). The aspect ratio $(=\mathrm{a} / \mathrm{b})$ of a prism is defined as the ratio of its height ('a') to twice the inradius of its polygonal face ('b'). The aspect ratio for HP and TP was set to 1 for which at close packing, the nearest neighbors of a particle have equal lattice vectors in all three directions. Moreover HP and TP of aspect ratio $1 / 2$ and 2 were also studied and the results presented as case studies A and B in the SI. Our model particles resemble the various inorganic and organic multi-faceted nano-colloidal particles that have been synthesized recently. ${ }^{23-26}$ The anisotropy is quantified using the asphericity values (ratio of the circumradius to the inradius) while the symmetry of the shapes is estimated by using the order of direct symmetry and a continuous parameter $^{22} q=1-\sum_{i<j}\left(\lambda_{i}^{2}-\lambda_{j}^{2}\right)^{2} /\left(\sum_{i} \lambda_{i}^{2}\right)^{2}$ that combines the eigen values (invariants) $\lambda_{i}$ of the particle inertia tensor $I_{i j}$. The asphericity values are $1.291,1.414,1.528,1.732$, 2.236 and 2.309 for TO, RD, HP, cubes, TP, and GB, respectively. The order of direct 
symmetry ${ }^{21}$ (number of distinct rotations reproducing the same orientation) is 24 for TO, $\mathrm{RD}$, and cubes, 12 for HP, 6 for TP, and 3 for GB; the continuous symmetry parameter $^{22} q$ is 1.000 for TO, RD, and cubes, 0.992 for HP, 0.967 for TP and 0.897 for GB. Clearly, the order of direct symmetry and the $q$ parameter rank the symmetry of the shapes in the same order but the latter is a more robust descriptor as small perturbations in particle geometry do not lead to abrupt changes in its value (e.g., for a distorted cube with one side $1 \%$ larger than the others, the $q$ value is decreased by less than $0.01 \%$ from the ideal value).

Extensive expansion and compression NpT MC runs for truncated octahedrons (TOs) and rhombic dodecahedrons (RDs) indicate the presence of a plastic crystalline mesophase for volume fractions in between the crystalline and isotropic phase regimes. The crystalline phases, which have a BCC structure for TO and FCC structure for RD (Fig. 2a and 2b), undergo continuous transitions to plastic crystalline (rotator) phases, which in turn melt into isotropic phases via first-order transitions. In the rotator phases (shown in Fig. 2a and 2b), the positional order is maintained but the short-ranged anisotropic orientation allows particles to rotate around their lattice positions.

The presence of appreciable hysteresis in compression and expansion runs precludes pinpointing therefrom the coexistence points for the isotropic-rotator phase transitions. Hence, we carried out additional direct interfacial $N v T$ simulations (details in Methods) which yield estimates of the coexistence densities as $\Phi_{\text {rotator }}=0.52$ and $\Phi_{\text {isotropic }}=0.47$ for TO and $\Phi_{\text {rotator }}=0.52$ and $\Phi_{\text {isotropic }}=0.48$ for RD. Using these values we plotted the 
equations of state shown in Fig. 3 using only the expansion run results for the crystal and rotator phase branches, and the compression run results for the isotropic phase branches. To identify the boundary for the rotator-crystal phase transition we devised an autocorrelation function for the orientation vectors of particles (details in Sec. 3.2 of $S /$ ), whose decay over simulation time (while using pseudo-dynamic MC moves) indicates loss of orientational order. The density at which this autocorrelation function starts decaying exponentially (as opposed to linearly, for higher densities) is marked as the approximate boundary for crystal-rotator transition, establishing $0.52<\Phi<0.70$ and $0.52<\Phi<0.68$ as the ranges for rotator phase stability for TO and RD, respectively.

To further characterize the structure of various phases, the orientational order in the system was measured using the cubatic order parameter $\left\langle\overline{P_{4}}\right\rangle^{13}$, while translational order was assessed via the bond-order orientational parameters $Q_{4}$ and $Q_{6}{ }^{27}$ (for ideal BCC packing $Q_{4}=0.036$ and $Q_{6}=0.511$, for ideal FCC packing $Q_{4}=0.191$ and $Q_{6}=0.575$ ). The plot in Fig. 3 suggests that while there is low orientational order in states between $\Phi \sim 0.52$ and $\Phi \sim 0.70$; the translational order is still maintained (high values of $Q_{6}$ ). For $T O$, the slightly off $Q_{4}$ values in this region suggests a disturbance of BCC order. This supports the idea that a reduced orientational entropic bonding field imparts sphere-like behaviour to the particles, whereby they maximize their packing entropy by reorganizing in structures akin to a HCP (ideal $Q_{4}=0.1$ ) or FCC (ideal $Q_{4}=0.19$ ) lattice (more stable for spheres ${ }^{28}$ ). To elucidate the structure of this intermediate mesophase, we followed Lechner et al. ${ }^{29}$ to obtain the distribution of averaged local bond order parameters $\overline{q_{4}}$ 
and $\overline{q_{6}}$ for various reference structures (BCC, FCC, HCP crystal lattices and liquid phase) and for the rotator structure obtained at $\Phi=0.594$ (details in Sec. 2.2 of $S /$ ). The fractions of each of these reference structures ${ }^{30}$ in the rotator phase of TO were found to be $f_{b c c}=0.001, f_{f c c}=0.140, f_{\text {hcp }}=0.859$ and $f_{\text {liq }}=0$, confirming the reorganization of the BCC structure. The snapshots of Fig. $2 a$ and $2 b$ show that the colours ascribed to different layers of the original $\mathrm{BCC}$ and FCC structures remain unperturbed for the rotator phases, illustrating preservation of translational order.

Simulations for hexagonal prisms (HPs) show existence of two different mesophases, a high density restricted plastic solid (RPS) and a cubatic-like liquid crystalline phase, in between the HCP crystal and the isotropic phase. The hysteresis effects across the transition points between expansion and compression runs in this system are rather small, hence coexistence densities for the cubatic to isotropic transition were estimated straightforwardly as $\Phi_{\text {cubatic }}=0.54$ and $\Phi_{\text {isotropic }}=0.49$. The RPS phase is similar to the "intermediate" phase that Blaak and Frenkel ${ }^{31}$ obtained for a system of hard cylinders, in which particles are either oriented along the director or are rotated in a plane normal to the director along one of the four-fold degenerate orientations (Fig. 2c). Of course, HP (and $n$-gonal prisms with $n>6$ ) are expected to approach the behaviour of hard cylinders of comparable aspect ratio at low-to-intermediate concentrations. The RPS phase has high translational order (solid-like) and discrete (six-fold) orientational disorder (or 'restricted' plastic character). On further expansion a cubatic-like phase is observed for a very narrow range of densities. While this phase also has a moderate amount of layering (as shown by the particle distribution functions shown in Fig. S5a), the particles 
have high translational mobility as indicated by the intermingling of their colours (assigned to layers in the original crystal, see Fig. 2c) and the moderate values of translational mobility coefficients in Fig. 4. The cubatic phase is only observed when using very small pressure changes in compression and expansion runs, which suggests that it could be a metastable intermediate state between the crystalline and isotropic phases.

We calculated the translational mobility coefficients $(D)$ and rotationally invariant orientational order parameters $I_{2}$ and $I_{4}{ }^{13,31}$ (details in $S I$ ) to mark the approximate boundaries of these transitions. Fig. 4 shows the equation of state (obtained by combining the expansion run for the meso- and crystalline phases, and compression run for the liquid phase) and the evolution of $I_{2}, I_{4}$, and $D$ with volume fraction. It can be observed that at $\Phi=0.54$ and 0.55 there are two first order transitions in the equation of state as well as transitions in both $I_{2}$ and $I_{4}$ values, which separate the isotropic, cubatic, and RPS phases. For $0.54<\Phi<0.55$ the small value of $I_{2}(\approx 0.1)$ compared to $I_{4}(\approx 0.3)$ is indicative of cubatic-like order; however, the modest $I_{4}$ value reveals a somewhat weak orientational alignment. Further, significantly higher values of mobility coefficients compared to those for the RPS phase indicate a dynamical translational disorder in this phase. For the $\Phi>0.62$ region, $I_{4}>0.8$ and $I_{2}>0.9$, reflecting development of perfect alignment between particles and hence a crystalline morphology. For the "crossover" region of $0.58<\Phi<0.62$, the values of $I_{2}$ and $I_{4}$ are intermediate and comparable, and $D$ values correspond to a solid-like phase; this combination of features defines the approximate boundaries for the RPS phase. Note that although cylinders showed a 
similar RPS phase, they did not exhibit a phase with cubatic order ${ }^{31}$ like the HPs. This difference can be attributed to packing entropy; a particle with an orientation perpendicular to its neighbors can pack its flat ends (top and bottom) more efficiently against the side facets of the prisms than against the round surface of cylinders.

Cubes have intermediate amount of anisotropy between HP and TP but the highest orientational symmetry (the same as TO). Cubes show a cubatic mesophase (Fig. 2d), which is bounded by a seemingly continuous transition from the simple-cubic crystalline phase (Fig. 2d) at higher densities and by a first-order transition from the isotropic phase at lower densities. The equation of state from expansion and compression runs is shown in Fig. 5 with phase boundaries marked by transitions in order parameter values. While cubes and other tetragonal parallelepipeds have already been studied extensively by John and Escobedo ${ }^{11-13}$, our new analysis reveals (see below) that early observations regarding the structure of the cubatic phase and the location of the cubatic-crystal phase boundary needed to be revisited.

The cubatic phase is a liquid crystalline phase characterized by high orientational order along all the three possible directors and by no long-range translational order. To mark the approximate boundaries of the two transitions, we measured the cubatic orientational order parameter $\left\langle\overline{P_{4}}\right\rangle$ and the mobility coefficients $D$. As observed in Fig. 5, the orientational order parameter $\left\langle\overline{P_{4}}\right\rangle$ shows a transition at $\Phi=0.52$ with high values for $\Phi>0.52$. The $D$ values are significantly higher for $0.52<\Phi<0.57$ compared to those 
in the crystalline region. Thus state points with $0.52<\Phi<0.57$ meet the criteria that define a cubatic phase, with crystalline phase stable for $\Phi>0.58$ and isotropic phase for $\Phi<0.45$. The higher rotational symmetry of cubes relative to hexagonal prisms may have played a role in stabilizing the cubatic phase over a comparatively wider range of densities. In contrast to earlier conjectures ${ }^{11-13}$, we now find clear evidence of layering in the cubatic phase (also observed in [32]) which becomes visible in our redefined particle distribution functions (see details in $S I$ ). While the finite size of our system prevents us from ascertaining the range of such a positional order, the appreciable $D$ values observed at these densities indicates that the system has liquid-like behaviour and there is sufficient translational disorder for layers to rearrange dynamically. This is corroborated by the intermingling of the colours ascribed to different layers of the original crystal in snapshots obtained at these densities (Fig. 2d). The interfacial simulations unveiled another peculiar behaviour: the interface between the isotropic and cubatic phases is very difficult to stabilize (leading to more interfaces as the simulation progresses), suggestive of a very low interfacial free energy.

The triangular prisms (TPs) and gyrbofastigiums (GBs) are distinctly anisotropic and the least symmetric shapes in the class of SFPs. Expansion runs for both of these shapes show a direct first-order melting transition and the absence of any mesophase between the crystalline solid and the isotropic phases. Further, compression runs failed to nucleate defect-free crystalline phases from the isotropic phase due to a large freeenergy barrier and non-ergodic configurational sampling. We determined the coexistence conditions for the isotropic-crystal phase transitions from direct simulation 
of the inhomogeneous systems ${ }^{33}$, composed of solid and liquid phases separated by an interface. The equations of state are plotted in Fig. 6a, by combining these coexistence density values with the expansion-run branches for $\Phi_{\text {solid }}>0.59$ and compression-run branch for $\Phi_{\text {liquid }}<0.47$ for TP and $\Phi_{\text {solid }}>0.62$ and $\Phi_{\text {liquid }}<0.49$ for GB.

The absence of a mesophase for TPs and GBs can be rationalized as follows. To form a mesophase with translational disorder in even one-direction (columnar-like phase), particles belonging to different columns would need to be able to move along their axes simultaneously. The particle interdigitation in the GB crystal, however, hinders the formation of a mesophase with partial spatial order. In the TP crystal, particles have a very low in-plane symmetry and even slight rotations about any of the axes would cause a large mismatch and disrupt the columnar structure (though it may be attainable for TPs of lower aspect ratios due to reduced losses in packing entropy along the short axis). A rotator mesophase is also precluded because rotation of particles out of the director's plane (layers) is restricted, as revealed by the orientational distribution function of one of the transverse axes for TP (see Fig. S6a) near the transition point ( $\Phi$ $=0.546$ ). Due to the low symmetry of TPs and GBs, the packing of the crystals is such that flipping of any particle to a perpendicular plane will require neighboring particles to be separated by a relatively large distance which would cause local melting and promote the isotropization transition, preempting formation of a plastic phase.

To better characterize the particle "flip-ability"' idea introduced above, we calculated the "flip factors" $\left(a_{f f i p}\right)$ by which the regular closed packing of each shape has to be 
expanded to allow one particle at its centre to flip to a perpendicular plane (Fig. 6b). These $a_{f l i p}$ values are 1.0, 1.0, 1.367, 1.0, 1.667 and 1.732 for TO, RD, HP, cubes, TP, and GB respectively. To get an estimate of the propensity for rotation of particles to locally melt the crystal, we used a Voronoi construction to calculate the average distances between neighboring particles $\left(a_{\text {liq }}\right)$ at their highest-density liquid states. The values for $a_{\text {liq }}$ obtained by averaging over several configurations are $1.41,1.37,1.46$, 1.58, 1.75 and 1.49 for TO, RD, HP, cubes, TP and GB, respectively. The ratios $a_{\text {flip }} / a_{\text {liq }}$ are consistent with the observed phase behaviours in that cubes, RD and TO can rotate normal to their lattice plane over a wide range of densities well above their isotropic densities, the range is very narrow for HP and TP, while it is less that 1 for GB precluding out-of-plane rotations for densities above their isotropic density.

We also calculated the expansion factors for free in-plane rotation of these particles $\left(a_{\text {planar }}\right)$, which should now be indicative of the ease with which particles form mesophases with in-plane rotational disorder like in-plane rotator phases or smectic phases. These $a_{\text {planar }}$ factors are $1.118,1.154,1.154,1.414,2.0$ and 1.414 for TO, RD, $\mathrm{HP}$, cubes, TP and GB, respectively. While the interdigitations at close packing of TO (and all Archimedean solids), RD (Catalan solids), and GB (Johnson solids) will prevent formation of liquid crystalline phases with partial spatial order (smectic and columnar phase), we expect and observe (see Ref. [13] and Case Studies A and B in the SI) formation of some of these phases for cuboids and HP and TP of certain aspect ratios. These phases arise from the disparity in translational and packing entropy values along different directions and hence they appear only at aspect ratios significantly greater or 
less than 1. Indeed, as density decreases, the growing amplitude of spatial vibrations in the crystal lattice will tend to destroy first translation order along the axis aligned with the smallest inter-particle spacing (thus producing a liquid crystal); this effect would be stronger for shapes with disparate dimensions (large asphericity).

The emerging picture as charted in Fig. $1 \mathrm{~b}$ is that: (i) shapes with low anisotropy and high orientational symmetry (TO and RD) are more likely to form plastic crystalline phases, (ii) shapes with intermediate anisotropy and high symmetry (cubes and HP of aspect ratio 1) are more prone to exhibit complex liquid crystalline mesophases; e.g., a cubatic-like phase, (iii) shapes with high anisotropy and low symmetry (GB and TP of aspect ratio 1) have no mesophase and show a direct isotropic-crystal phase transition, and (iv) sufficiently oblate prisms exhibit columnar phases while sufficiently prolate prisms can exhibit smectic phases. Additionally, any shape in the limit of aspect ratio tending to zero or infinity are expected to approach the behaviour of disks or long rods which exhibit nematic phases. Other SFPs ${ }^{34-35}$ and polyhedral particles are expected to conform with these qualitative trends.

Very high hysteresis is observed across the first order transition from the liquid to translationally ordered phases for TO, RD, GB, and TP, while negligible hysteresis is seen for transitions from liquid to orientationally ordered phases for HPs and cubes. This suggests that it may always be easier to nucleate (lower free energy barrier) orientational order than translational order from a liquid phase. Also, when a mesophase occurs, the mesophase-crystal transition appears to be continuous, a 
rather remarkable result for a cubatic phase. As illustrated by the case of TOs, a rotator phase for any particle with low anisotropy and high rotational symmetry may always have translational order with dominant HCP/FCC symmetry, irrespective of the native densest-packing lattice (Bravais for centrally symmetric polyhedrons ${ }^{17}$ ). Regarding the cubatic phases observed (for cubes and HP), their significant layering underscores the importance of distinguishing between static vs. dynamic order. Indeed, our calculations of mobility coefficients indicate that "time" or dynamics is a key consideration in characterizing the liquid crystalline character of phases that otherwise possess a significant degree of 'static' positional order. While 2D ordered assemblies have been experimentally observed for cubes ${ }^{36}$ and $\mathrm{HP}^{37}$, experimental verification of the various structures predicted in this study is yet to be achieved.

\section{Methods}

For outlining the phase behaviour for each of the SFP shapes under study, extensive isothermal-isobaric NpT ensemble Monte Carlo simulations were performed. The number of particles used in these simulations depended on the geometry of the packing specific to particle shape, such that a perfect crystal (space filling) configuration can be obtained to start the simulation for expansion runs. To account for finite size effects, we simulated systems of 1024, 2000 and 3456 particles for each of the particle shapes except for cubes for which we used 512, 1000 and 1728 particles and for GB for which we used 2916 particles. The methodology involved equilibrating the system at a particular osmotic pressure $P^{*}$ using translation, rotation and anisotropic volume moves. 
The dimensionless osmotic pressure $P^{*}$ was defined as $P^{*}=P a^{3} / \varepsilon$, where ' $a$ ' is the radius of the circumscribing sphere (specific to each particle geometry) and ' $\varepsilon$ ' is an arbitrary energy parameter (set to 1 ). We also incorporated "flip moves" ${ }^{31}$, that attempt to rotate a chosen particle to a random orientation in the plane perpendicular to its current orientation. All moves were accepted or rejected according to the Metropolis criterion which in this case involves overlap checks using the separating axes theorem ${ }^{38}$.

Expansion/compression runs were performed in a stepwise manner by expanding the perfect solid crystal structure at decreasing/increasing values of pressure. The volume moves were anisotropic to allow the simulation box shape to change for the solid-phase and mesophase branches, while only isotropic volume moves were allowed at pressures corresponding to the liquid region (to avoid highly distorted box shapes). Each pressure run consisted of $3 \times 10^{6}$ Monte Carlo cycles for both equilibration and production, where each cycle has on average $N$ translational, $N$ rotational, $N / 10$ flip, and 1 volume move attempts. Because of the large free energy barriers, nucleation of an ordered phase from an isoptropic phase required longer and slower compression runs. Hence, smaller pressure steps size and more numerous MC cycles (up to $2 \times 10^{7} \mathrm{MC}$ cycles) were often used. The equilibration of each phase was checked by recording the volume of the simulation box against the number of cycles and by the consistency of various order parameters measured at key state points for configurations periodically saved during the production runs. In case of appreciably hysteresis across a first order transition, direct interfacial $N v T$ simulations were carried out to get the coexistence densities of the two phases. The initial configuration for the two-phase interfacial system 
was built using volume fractions at the transition points obtained from expansion runs (as initial estimates). At the start, the two boxes with perfect crystalline phases are placed next to each other, the second region is further expanded along the z-axis to match the lower density of the liquid region. Following this, the liquid region and solid regions are separately equilibrated using $2 \times 10^{7} \mathrm{MC}$ cycles each. The resulting heterogeneous system was then equilibrated with $3 \times 10^{7} \mathrm{MC}$ cycles to obtain an equilibrated two-phase state. The coexistence densities are calculated by plotting the particle density along the $z$ axes and using the plateau region of this density profile for the two phases. All phases obtained in this study were further analyzed using various translational and orientational order parameters, mobility coefficients, plots for orientational distribution functions, particle distribution functions, and visual inspection of snapshots at all state points. A detailed account of these methods is given in S/ along with some additional plots.

Correspondence and requests for materials should be addressed to F.A.E.

\section{Acknowledgments}

This work was supported by a Department of Energy Basic Energy Science Grant ER46517. This publication is based on work supported in part by award no. KUS-C1018-02, made by King Abdullah University of Science and Technology (KAUST).

\section{Author contributions}

U.A. and F.A.E. planned the research, analysed the data, and wrote/revised the manuscript. U.A. carried out the simulations. 


\section{References}

1. Whitesides, G.M. \& Boncheva, M. Beyond molecules: Self-assembly of mesoscopic and macroscopic components. Proc. Natl. Acad. Sci. 99, 4769-4774 (2002).

2. Glotzer, S.G. \& Solomon, M.J. Anisotropy of building blocks and their assembly into complex strucures. Nat. Mater. 6, 557-562 (2007).

3. Frenkel D. Soft Condensed Matter. Physica A 313, 1-31 (2002).

4. Burda C., Chen X., Narayanan R. \& El-Sayed M. Chemistry and properties of nanocrystals of different shapes. Chem. Rev. 105, 1025-1102 (2005).

5. Tuteja A., Mackay M.E., Narayanan S., Asokan S. \& Wong M.S. Breakdown of the continuum Stokes-Einstein relation for nanoparticle diffusion. Nano. Lett. 7, 1276-1281(2007).

6. Gudiksen M.S., Lauhon L.J., Wang J., Smith D.C. \& Lieber C.M. Growth of nanowire superlattice strucures for nanoscale photonics and electronics. Nature 415, 617-620 (2002).

7. Lee Y.S., Wetzel E.D. \& Wagner N.J. The ballistic impact characteristics of kevlar woven fabrics impregnated with a colloidal shear thickening fluid. J. Mater. Sci. 38, 2825-2833 (2003).

8. Veerman J.A.C. \& Frenkel D. Phase-behavior of disk-like hard-core mesogens. Phys. Rev. A 45, 5632-5648 (1992).

9. Camp P.J. \& Allen M.P. Phase diagram of the hard biaxial ellipsoid fluid. J. Chem. Phys. 106, 6681-6689 (1997). 
10. Zhang Z., Tang Z., Kotov N.A. \& Glotzer S.C. Simulations and analysis of selfassembly of CdTe nanoparticles into wires and sheets. Nano. Lett. 7, 1670-1675 (2007).

11. John B.S., Stroock A. \& Escobedo F.A. Cubatic liquid-crystalline behavior in a system of hard cuboids. J. Chem. Phys. 120, 9383-9389 (2004).

12. John B.S. \& Escobedo F.A. Phase behavior of colloidal hard tetragonal parallelopipeds (cuboids): A Monte Carlo simulation study. J. Phys. Chem. B 109, 23008-23015 (2005).

13. John B.S. \& Escobedo F.A. Phase behavior of colloidal hard perfect tetragonal parallelopipeds. J. Chem. Phys. 128, 044909 (2008).

14. Yin J.S. \& Wang Z.L. Ordered Self-Assembly of Tetrahedral Oxide Nanocrystal. J. Chem. Phys. 129, 114707 (1997).

15. Maeda H. \& Maeda Y. Liquid crystal formation in suspensions of hard rodlike colloidal particles: direct observation of particle arrangement and self-ordering behavior. Phys. Rev. Lett. 90, 018303. (2003).

16. Jiao Y., Stillinger F.H. \& Torquato S. Optimal packing of superballs. Phys. Rev. E 79, 041309 (2009).

17. Torquato S. \& Jiao Y. Dense packings of the platonic and archimedean solids. Nature 460, 876-879 (2009).

18. Steinhaus H. Mathematical Snapshots. (Oxford Univ. Press, New York, 1999).

19. Chen E. R., Engel M. \& Glotzer S. C. Dense crystalline dimer packings of regular tetrahedral. Discrete Comput. Geom. 44, 253-280 (2010). 
20. Torquato S. \& Jiao Y. Exact constructions of a family of dense periodic packings of tetrahedral. Phys. Rev. E 81, 041310 (2010).

21. Miller W. Symmetry Groups and their Applications. (Academic Press, New York, 1972).

22. Miller W. L., Bozorgui B. \& Cacciuto A. Crystallization of hard aspherical particles. J. Chem. Phys. 132, 134901 (2010)

23. Barnard A.S., Lin X.M. \& Curtiss L.A. Equilibrium morphology of face-centred cubic gold nanoparticles $>3 \mathrm{~nm}$ and the shape change induced by temperature. J. Phys. Chem. B 109, 24465-24472 (2005).

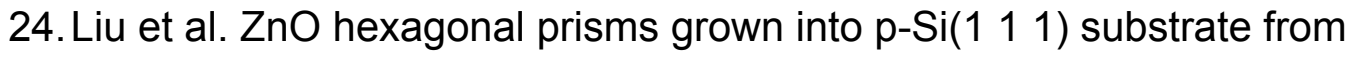
poly(vinylpyrrolidone) assisted electrochemical assembly. J. Crys. Growth 290, 405-409 (2006).

25. Sun Y.G. \& Xia Y.N. Shape-controlled synthesis of gold and silver nanoparticles. Science 298, 2176-2179 (2002).

26. Millstone J.E., Hurst S.J., Mètraux G.S., Cutler J.I. \& Mirkin C.A. Colloidal gold and silver triangular nanoprisms. Small 5, 646-664 (2009).

27. Steinhardt P.J., Nelson D.R. \& Ronchetti M. Bond-orientational order in liquids and glasses. Phys. Rev. B 28, 783-805 (1983).

28. Mau S.C. \& Huse D.A. Stacking entropy of hard-sphere crystals. Phys. Rev. E 59, 4396-4401 (1999).

29. Lechner W. \& Dellago C. Accurate determination of crystal structure based on averaged local bond order parameters. J. Chem. Phys. 129, 114707 (2008). 
30. ten Wolde PR, Ruiz-Montero MJ, Frenkel D Numerical evidence for bcc ordering at the surface of a critical fcc nucleus. Phys. Rev. Lett. 75, 2714-2717 (1995).

31. Blaak R., Frenkel D. \& Mulder B.M. Do cylinders exhibit a cubatic phase? J. Chem. Phys. 110, 11652-11659 (1999).

32. Batten R.D., Stillinger F.H. \& Torquato S. Phase behavior of colloidal superballs: Shape interpolation from spheres to cubes. Phys. Rev. E 81, 061105 (2010).

33. Noya E.G., Vega C. \& Miguel E. Determination of the melting point of hard spheres from direct coexistence simulation methods. J. Chem. Phys. 128, $154507(2008)$

34. Inchbald G. Five space-filling polyhedral. The Mathematical Gazette 80, 466 (1996).

35. Goldberg M. Several new space-filling polyhedral. Geometriae Dedicata 5, 517 (1976).

36. Choi S.Y., Lee Y.J., Park Y.S., Ha K. \& Yoon K.B. Monolayer assembly of zeolite crystals on glass with fullerene as the covalent linker. J. Am. Chem. Soc. 122, 5201-5209 (2000).

37.Zhang H.T., Wu G. \& Chen X.H. Large-scale synthesis and self-assembly of monodisperse hexagon Cu2S nanoplates. Langmuir 21, 4281-4282 (2005).

38. Golshtein E.G. \& tretyakov NV Modified Lagrangians and monotone maps in optimization. (Wiley, New York, 1996). 
a

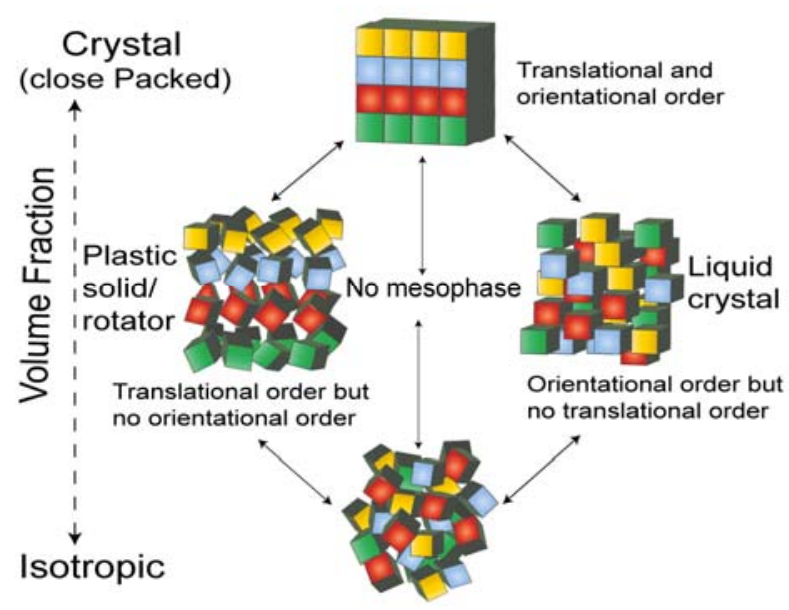

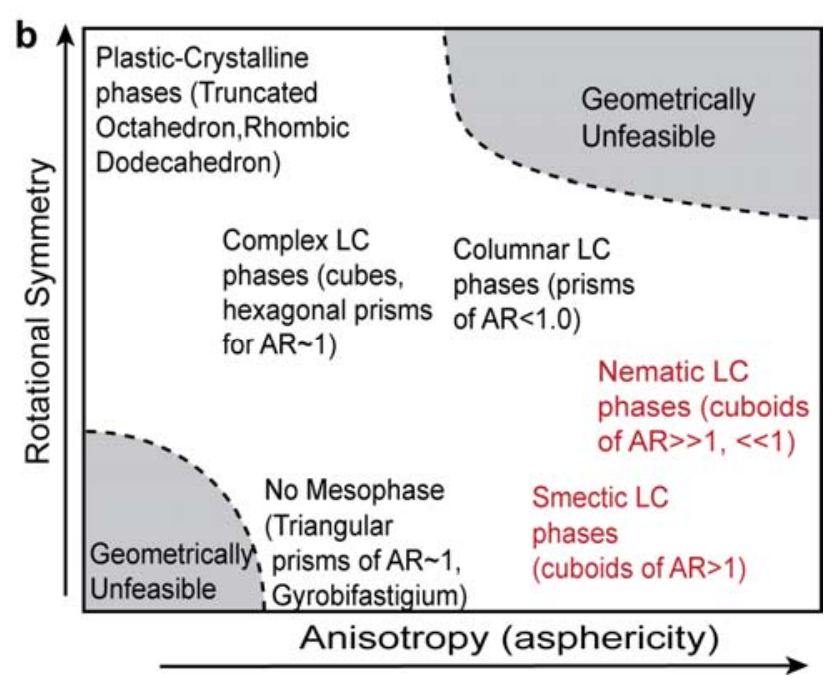

Figure 1. Effect of shape anisotropy on hard-particle mesophase order. (a)

Possible states at intermediate volume fractions; partial loss of order in particular degrees of freedom in SFP crystals can lead to mesophases. (b) Approximate roadmap for phase behaviour of hard polyhedral shapes. $L C=$ liquid crystal, $A R=$ aspect ratio; the phases shown in red are expected to be stable for prisms of similar AR. 


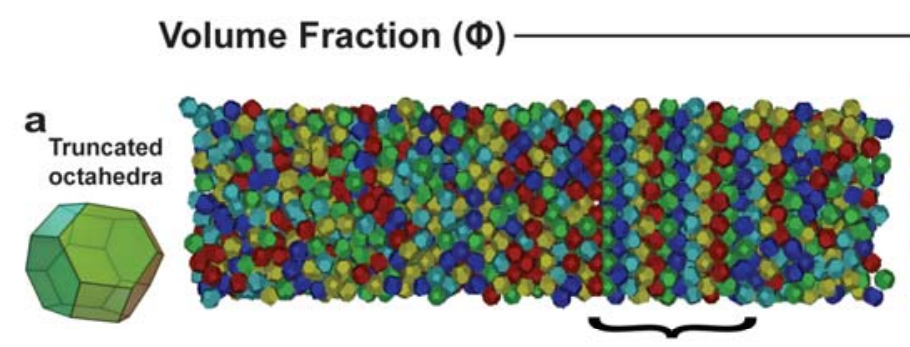

Coexistence: Isotropic $(\Phi=0.47)+$ Rotator $(\Phi=0.52)$
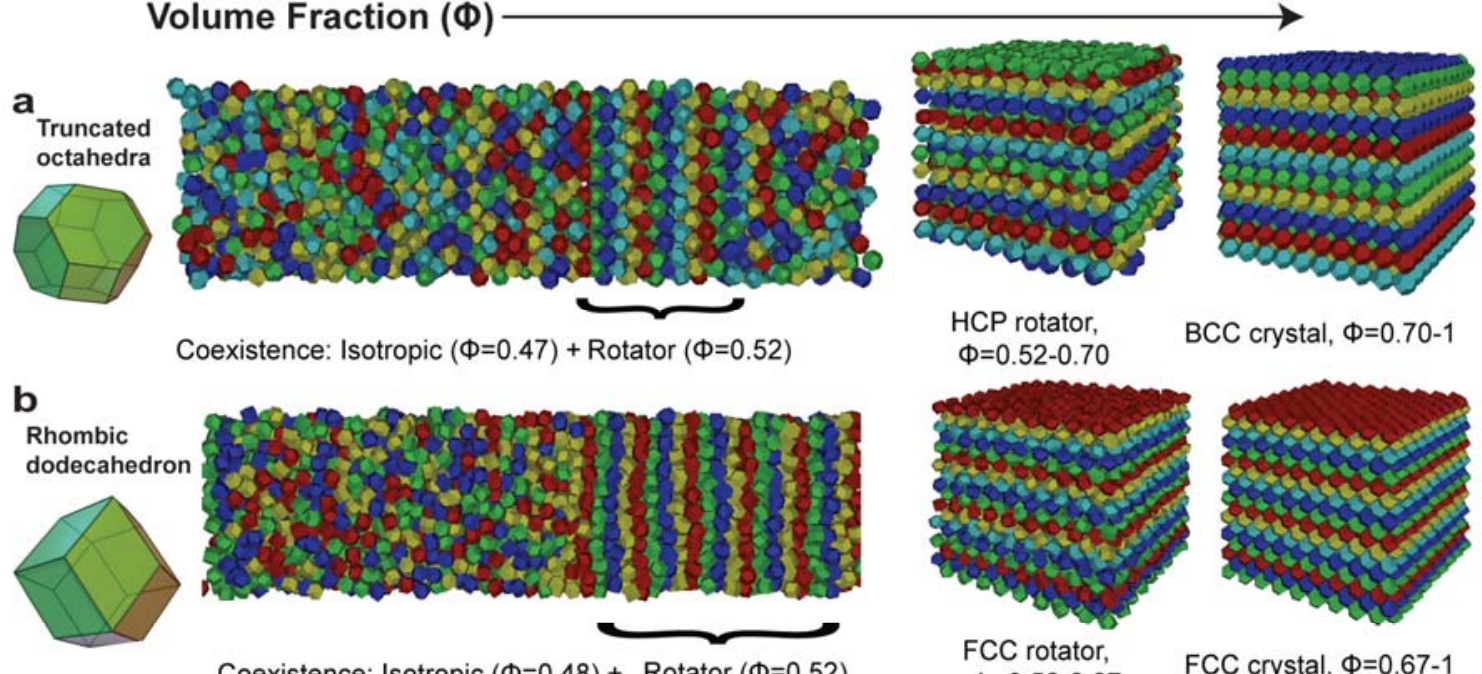

를

HCP rotator, $\Phi=0.52-0.70$

BCC crystal, $\Phi=0.70-1$
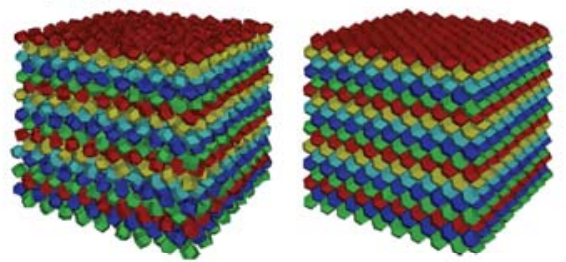

FCC rotator, $\Phi=0.52-0.67$

FCC crystal, $\Phi=0.67-1$

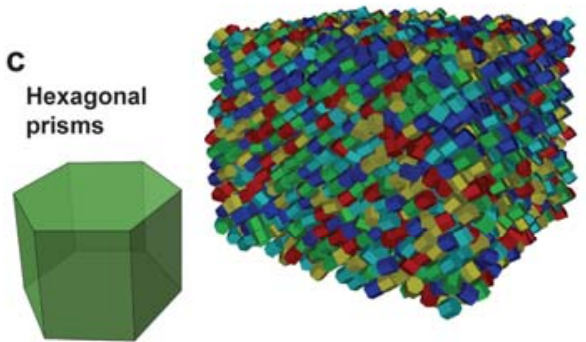

Quasi cubatic, $\Phi=0.54-0.55$

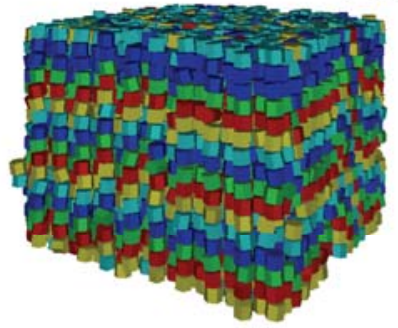

Plastic, $\Phi=0.58-0.62$

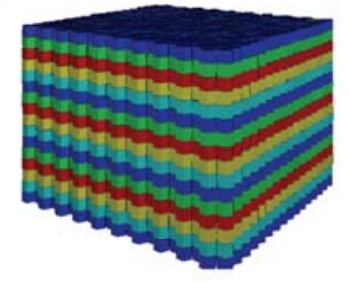

HCP crystal, $\Phi=0.63-1$

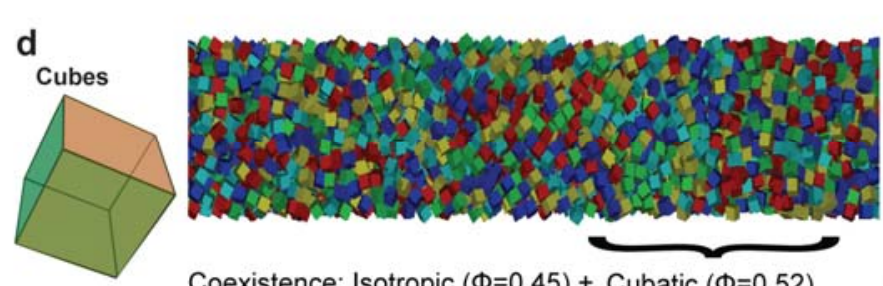

Coexistence: Isotropic $(\Phi=0.45)+$ Cubatic $(\Phi=0.52)$

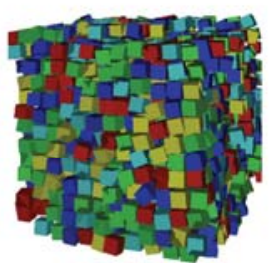

Cubatic, $\Phi=0.52-0.57$

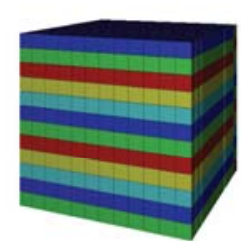

SC crystal, $\Phi=0.58-1$

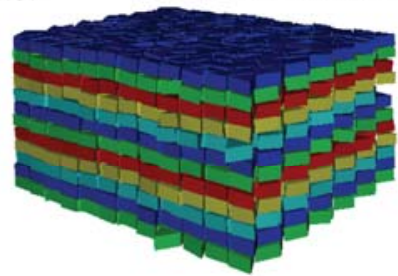

Honeycomb crystal, $\Phi=0.59-1$

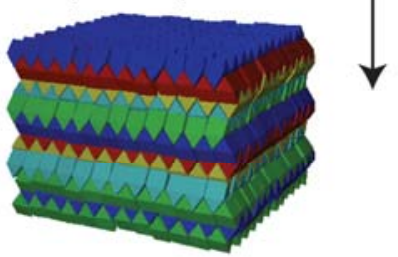

ABCD cubic crystal, $\Phi=0.62-1$ 
Figure 2. Summary of equilibrium structures for different SFPs. Snapshots for the various mesophases, and crystalline states for the six shapes (a) Truncated Octahedrons, (b) Rhombic Dodecahedron, (c) Hexagonal Prisms, (d) Cubes, (e) Triangular Prisms and (f) Gyrobifastigiums, obtained from expansion and coexistence runs. The states are depicted from left to right in increasing order of volume fractions and the six shapes are arranged from top to bottom in increasing order of anisotropy. Particle colours are used for visual effect and to detect mixing of the initial crystal layers.

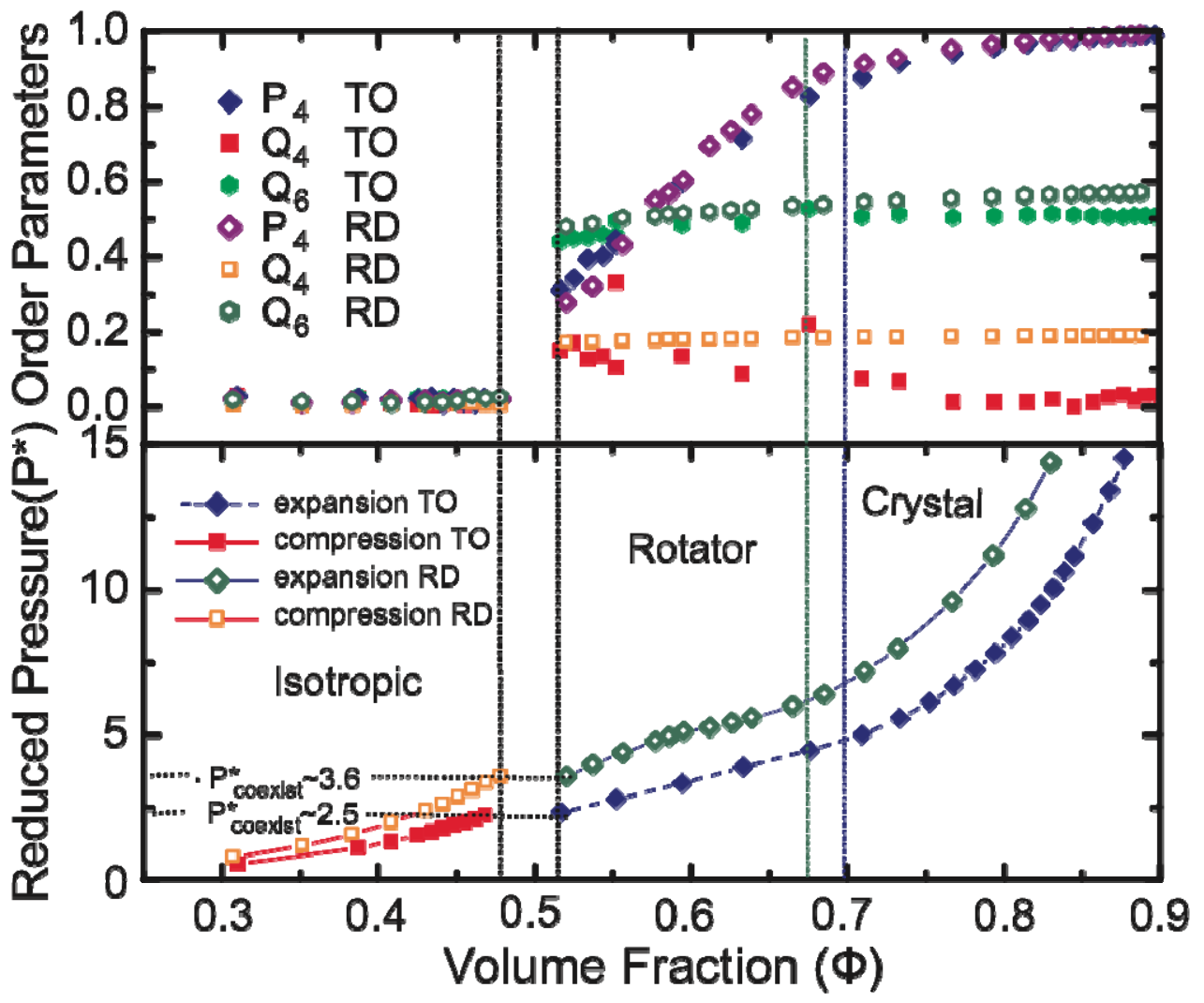

Figure 3. Phase behaviour of shapes with low anisotropy and high symmetry.

Translational $\left(Q_{4}\right.$ and $\left.Q_{6}\right)$ and orientational $\left(\left\langle\overline{P_{4}}\right\rangle\right)$ order parameters (top) and equation 
of state (bottom) with marked phase boundaries for truncated octahedrons (TO) and rhombic dodecahedrons (RD).

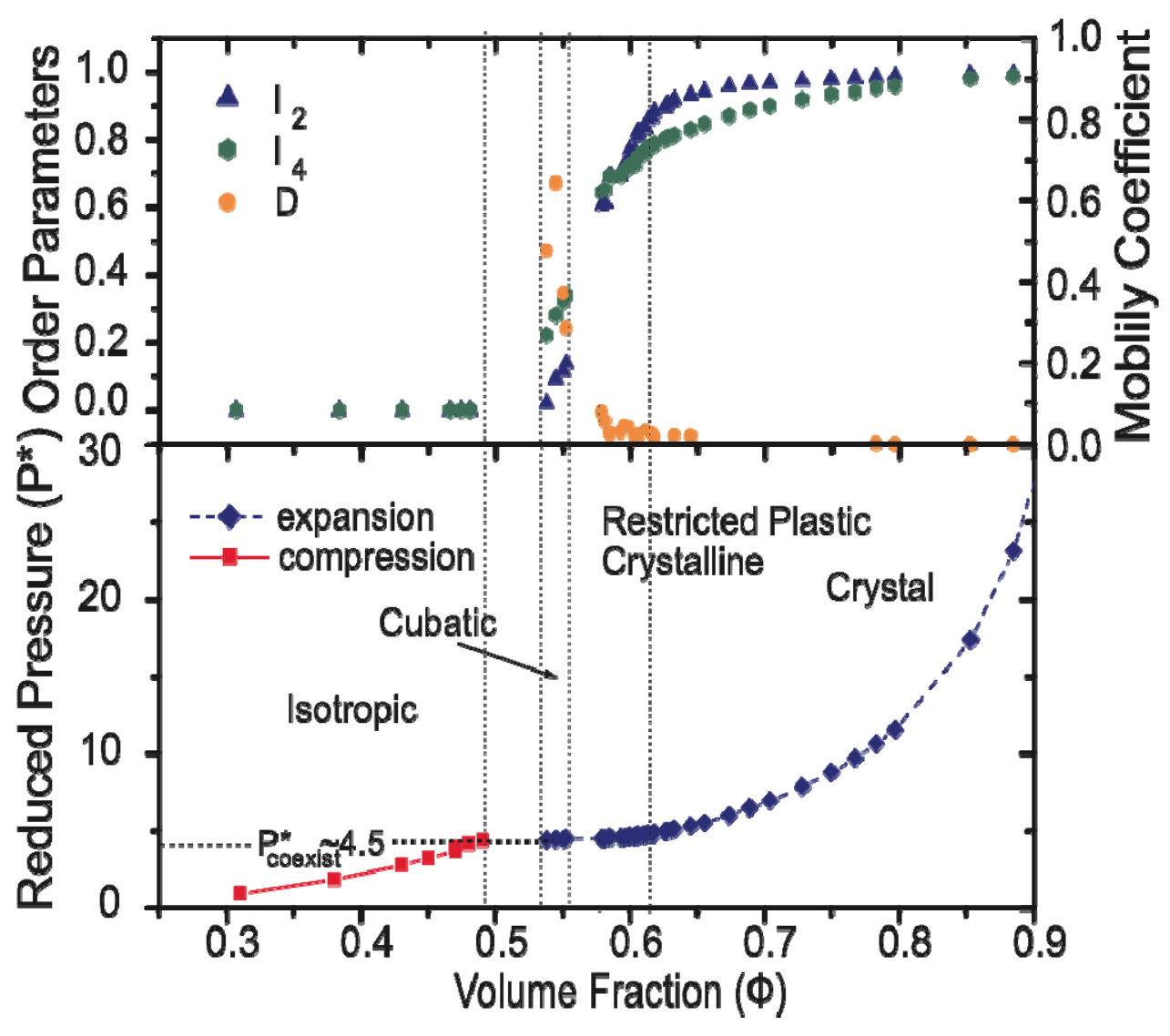

Figure 4. Phase and structural behaviour of hexagonal prisms. Translational mobility coefficient $D$, orientational order parameters $I_{2}$ and $I_{4}$ (top) and equation of state (bottom) with marked phase boundaries for hexagonal prisms. 


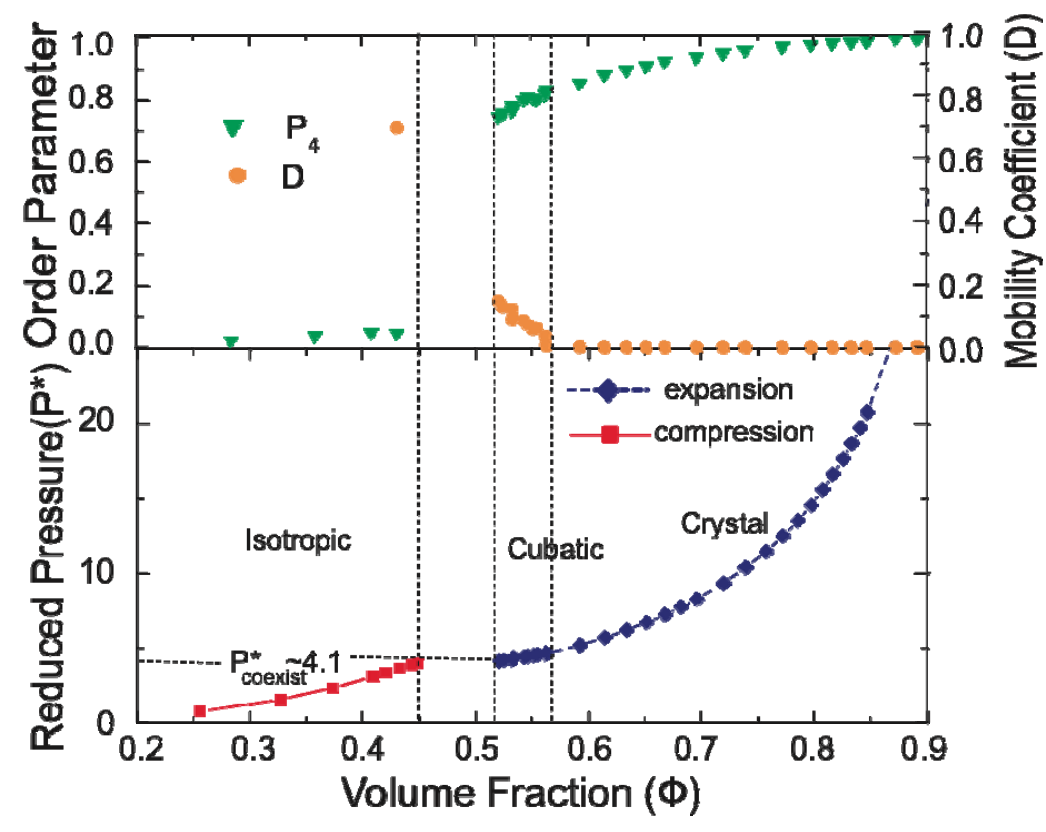

Figure 5. Phase and structural behaviour of cubes. Translational mobility coefficient $D$, orientational order parameter $\left\langle\overline{P_{4}}\right\rangle$ (top) and equation of state (bottom) with marked phase boundaries for cubes.
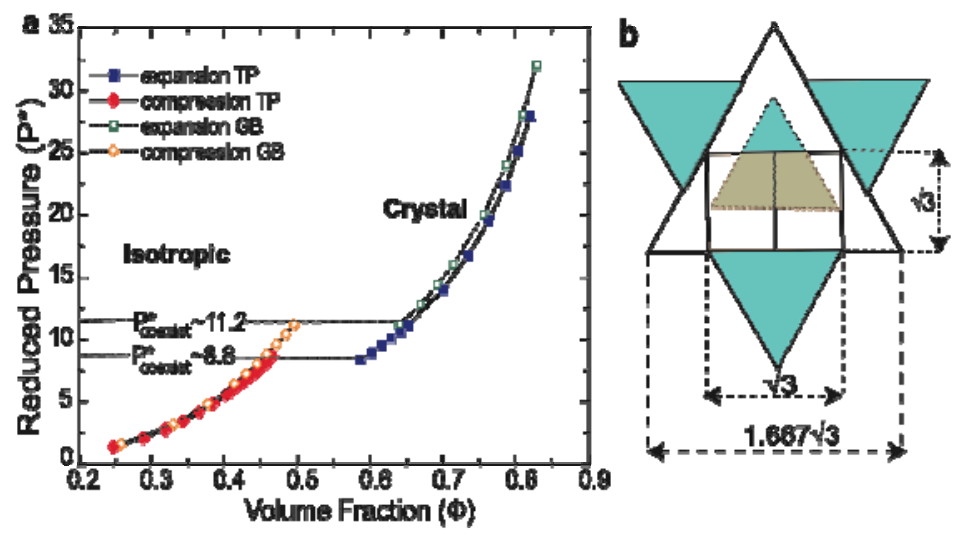

Figure 6. Phase behaviour for shapes with high anisotropy and low symmetry. a)

Equation of state curves for triangular prisms (TP) and gyrobifastigium (GB); (b) Illustration of the geometrical calculations for the degree of expansion required for regular packing to accommodate rotation of a particle (a TP in this case) at its centre. Polygon with dotted lines correspond to the original orientation of the central particle. 